AperTO - Archivio Istituzionale Open Access dell'Università di Torino

\title{
Fruitio et beatitudo entre volonté et intellect selon Pierre de Ceffons
}

\section{This is the author's manuscript}

Original Citation:

Availability:

This version is available http://hdl.handle.net/2318/1580036

since 2021-01-26T12:44:31Z

Published version:

DOI:http://dx.doi.org/10.1484/J.QUAESTIO.5.108644

Terms of use:

Open Access

Anyone can freely access the full text of works made available as "Open Access". Works made available under a Creative Commons license can be used according to the terms and conditions of said license. Use of all other works requires consent of the right holder (author or publisher) if not exempted from copyright protection by the applicable law. 


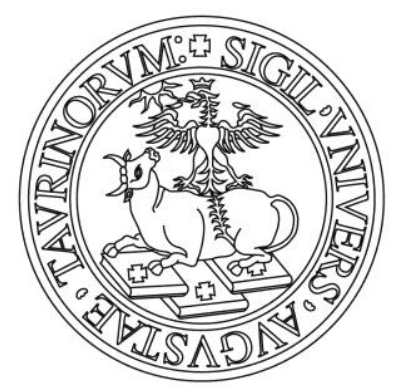

\section{UNIVERSITÀ DEGLI STUDI DI TORINO}

This is an author version of the contribution published on:

Questa è la versione dell'autore dell'opera:

\section{Amos Corbini}

Fruitio et beatitudo entre volonté et intellect selon Pierre de Ceffons QUAESTIO, 2015, 15, 721-728

The definitive version is available at: La versione definitiva è disponibile su: 
SUMMARY: in the sixteenth question of his commentary to the Sentences (1348-49), the Cistercian Peter of Ceffons shows sometimes an hesitating attitude towards the problems related to fruitio and beatitudo, sometimes instead a greater firmness, especially when other Parisian theologians of his time displayed a good degree of agreement. In doing so, he quotes explicitly English authors of the years 1320-30, but also precedings auctoritates like Thomas Aquinas and John Duns Scotus, and typical themes of discussion of the Oxford calculatores; moreover, only sometimes he agrees with his confrère John of Mirecourt.

KEYWORDS: happiness, beatitude, fourteenth century, English thought, John of Mlrecourt, Thomas Aquinas, Augustinian theology.

La seizième question du premier livre du commentaire aux Sentences du cistercien Pierre de Ceffons, rédigé à Paris en $1348-49^{1}$, pose le problème de savoir si la fruitio est un acte de la volonté ${ }^{2}$; il s'agit de la dernière d'un ensemble de cinq questions (allant de la 12 à la 16 pour être précis) où l'auteur affronte des problèmes liés à la première distinction du premier livre des Sentences.

Certes le problème ainsi posé semble plutôt délicat pour De Ceffons. C'est tout du moins ce qu'indique l'auteur dans la prémisse ${ }^{3} \mathrm{~d}^{\prime}$ une discussion dialectique sur le sujet où il reconnaît que certains pourraient penser que le problème est oisif (frivolum) du fait qu'en réalité l'intellect et la volonté sont une seule et même chose et qu'il n'est donc pas raisonnable de vouloir savoir si l'un a plus de valeur que l'autre. Il était notoire, en effet, que des auteurs anglais que Pierre connaissait avaient avancé des théories tendant à ne pas distinguer entre elles les facultés de l'âme, et les facultés de l'âme même, en posant en effet une unité absolue de l'âme qui donnerait lieu à des distinctions seulement opératives ${ }^{4}$. En acceptant cette idée, il pourrait donc sembler inutile d'établir une priorité entre intellect et volonté dans l'acte de la fruitio.

Mais à ce point, ajoute l'auteur, on pourrait aussi interpréter cette question en lui donnant une signification positive qui reviendrait à se demander si l'âme toute entière est plus noble quand elle exerce

\footnotetext{
${ }^{1}$ Sur la vie, les œuvres et certains éléments intéressants de sa spéculation, voir avant tout l'article fondamental de TRAPP 1957 et celui de TRAPP 1984. D’autres études sur des aspects spécifiques de la pensée de Pierre sont: MURDOCH 1978; BotTIn 1982, pp. 142-156; Genest 1984; TACHAU 1988, pp. 372-377; SMITH 1990; MAZET 2004.

${ }^{2}$ Utrum frui sit actus voluntatis (Petrus de CeffonA, In IV libros Sententiarum, ms. Troyes, BM 62, f. 36vb-42va).

${ }^{3}$ Ms. Troyes, BM 62, ff. 36vb-37ra.

${ }^{4}$ Nous trouvons ces discussions par exemple dans les commentaires de Walter Chatton, Richard FitzRalph et Adam Wodeham ; sur cela, voir REINA 1986, pp. 30-32 ; TACHAU 1988, pp. 240-241, 280-281 et 365.
} 
sa fonction intellectuelle ou quand elle accomplit un acte de volition; cela équivaut, dit Pierre, à se demander, secundum imaginationem, si l'intellect et la volonté sont effectivement des facultés de l'âme séparées les unes des autres ${ }^{5}$. Cette possibilité permet à Pierre d'établir trois conclusiones: dans la première, il conclut que la volonté est, somme toute, supérieure à l'intellect, bien que dès le début, il semble avoir beaucoup d'hésitations. II dit par exemple, eu égard à cette conclusion, qu'on pourrait

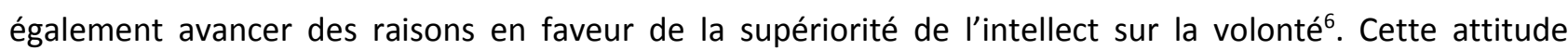
prudente se fait sentir dans l'argumentation de Pierre : la volonté peut être considérée comme supérieure parce qu'elle est plus élevée que l'intellect, parce qu'elle conduit l'intellect à choisir l'action à accomplir, ou bien encore parce que la charité, qui est la disposition acquise par la volonté, est meilleure que la sagesse, qui est la disposition acquise par l'intellect ${ }^{7}$. Et lorsque Pierre, après avoir énoncé les deux autres conclusions de sa question, revient aux objections qu'on pourrait adresser à cette première conclusion, il ajoute aussitôt que Thomas d'Aquin ne serait pas d'accord sur ce point ${ }^{8}$; il exprime ensuite une autre raison essentielle de ses hésitations sur les rapports entre volonté et intellect : on ne peut pas dire que le bonheur réside entièrement dans un acte de l'intellect parce que, pour être heureux, plusieurs choses sont nécessaires comme avoir des habits et faire des actions vertueuses jugées par la volonté. D'autre part, comme le dit Aristote en soutenant que le bonheur réside dans l'activité intellectuelle, la contemplation philosophique ne serait pas digne de louanges si elle n'était pas le résultat d'un choix de la volonté ${ }^{\text {. Mais }}$ encore une fois, Pierre, d'une allure quelque peu ondoyante, semble ensuite accorder à ses adversaires une part de raison : aussi admet-il, en effet, qu'il est vrai que parfois ceux qui savent ce qu'il est préférable de faire parviennent à le faire plus facilement ou, tout du moins, peuvent avoir de meilleurs habitus dans leur volonté ${ }^{10}$.

\footnotetext{
${ }^{5}$ Sur le sens d'imaginatio dans le commentaire de Pierre, je me permets de renvoyer à CORBINI 2013.

${ }^{6}$ Ms. Troyes, BM 62, f. 37vb.

${ }^{7}$ Ms. Troyes, BM 62, f. 37ra.

${ }^{8}$ Ms. Troyes, BM 62, f. 37rb-va.

${ }^{9}$ Ms. Troyes, BM 62, f. 37va.

${ }^{10}$ Ms. Troyes, BM 62, f. 37vb. On peut remarquer en passant que Pierre cite six fois explicitement dans cette question l'hibernicus Richard FitzRalph, toujours en rapport au problème posé dans la première conclusion. Pierre en effet indique à deux reprises, vers le début de sa question, son opinion en faveur de la supériorité de la volonté sur l'intellect (f. 37ra); il le refait encore par deux fois sur le même thème, lors de son questionnement dialectique (I'une des deux fois étant pour réfuter ses arguments, f. 37va) ; les deux dernières fois, FitzRalph est la source qu'utilise Pierre pour connaitre le passage augustinien où il est dit que l'intellect serait supérieur au sens parce qu'il juge l'opération du sens, et FitzRalph discute la possibilité de tirer de cette idée la conclusion que l'intellect est supérieur à la volonté aussi ; mais Pierre manifeste à cet égard beaucoup de réserves (f. 37vb).
} 
Inversement, Pierre est plus déterminé sur le deuxième point de son exposé, c'est-à-dire sur le fait que la fruition est sûrement un acte de la volonté. En effet, sur ce point Pierre pouvait s'appuyer sur une discussion très large ayant abouti à une concordance fondamentale chez les théologiens augustiniens et cisterciens ayant lu les Sentences à Paris dans les années précédentes ${ }^{11}$. C'est ainsi que, sur ce point, il s'aligne sur les thèses soutenues par Grégoire de Rimini et partagées par Alphonse Vargas et Hugolin d'Orvieto et que, dans les raisons évoquées brièvement dans sa thèse, il ne se réfère jamais au problème de savoir si la fruition pourrait être aussi un acte de l'intellect ${ }^{12}$. Et lorsque Pierre expose les raisons contra sa conclusion, il touche seulement à des problèmes spécifiques à l'activité et à la liberté de la volonté, jamais à son rapport avec l'intellect ${ }^{13}$. Pouvant donc s'appuyer sur une tradition claire, univoque, mais

11 Trapp avait envisagé en effet l'existence d'une grande cohérence doctrinale dans ce qu'il a appelé une « table ronde " de théologiens qui aurait réuni, avant Pierre, Grégoire de Rimini, Alphonse Vargas de Tolède, Hugolin d'Orvieto et Jean de Mirecourt (TRAPP 1984). Or, si cette cohérence doctrinale ne semble pas toujours si absolue et univoque (je me permets de renvoyer sur ce sujet à mon "Notitia intuitiva and complexe significabile in the Fifth Decade of the Fourteenth Century in Paris. From Alphonsus Vargas Toletanus to Peter Ceffons", à paraitre dans les actes du congrès SIEPM 2010), sur le point qui nous intéresse à présent, la thèse de Trapp, au contraire, semble valable : Grégoire établit en effet que n'importe quel acte de fruitio est un acte de la seule volonté, il l'argumente très rapidement et n'y revient plus (GREGORII ARIMINENSIS OESA Lectura super primum et secundum Sententiarum, ed. D. Trapp / V. Marcolino, t. I, p. 240 II. 7-8). Alphonse en arrive lui aussi à la même conclusion, tout en n'évitant pas tout à fait la discussion sur ce point, s'élevant en particulier contre l'opinion de Landolphe Caracciolo (ALPHONSI VARGAS TOLETANI OESA In primum Sententiarum, ed. Venetiis 1490, q. 3, col. 162-166). Hugolin, pour sa part, reste fidèle à la doctrine de Grégoire, en la proposant sans problèmes. Il se révèle toutefois plutôt critique à l'égard du maître augustinien sur le problème de la nature de l'acte de connaissance, qui aurait été nécessairement présupposé par celui de la volonté dans la fruition. II se situe sur ce point, somme toute, plus près de Adam de Wodeham, que de Grégoire qui lui l'avait critiqué (ce qui, soit dit en passant, n'est pas sans intérêt pour quelqu'un comme Hugolin, théologien assez conservateur; voir HUGOLINI DE URBE VETERI OESA Commentarius in quattuor libros Sententiarum, ed. W. Eckermann, t. I, pp. 188-200; GREGORII ARIMINENSIS OESA Lectura super primum et secundum Sententiarum, ed. D. Trapp / V. Marcolino, t. I, pp. 212-217). En ce qui concerne Jean de Mirecourt, il faut dire que les questions allant de 13 à 16 éditées par Massimo Parodi (PARODI 1977 et 1978) se présentent comme un vrai bon exemple de sa façon typique et quelque peu cryptique, de procéder; par conséquent je ne saurais indiquer dans leur texte une prise de position claire à ce sujet, à part quelques brèves allusions ici et là. Toutefois, dans la deuxième conclusion de la question 19 Jean semblerait pencher lui aussi du côté de ses contemporains parisiens, il ne présentera pas de discussion qui la remette en doute et n'utilisera aucune de ses typiques expressions restrictives (ms. Torino, BN, D. IV. 28, f. $45 \mathrm{vb}$ ) ; toutefois, il est nécessaire d'observer une certaine prudence sur la réelle signification de ce passage.

12 II aborde uniquement le problème de l'activité de l'âme humaine dans la fruition même, en poursuivant probablement la discussion dont Alphonse Vargas nous a précieusement témoigné (PETRUS DE CEFFONA, In IV libros Sententiarum, ms. Troyes, BM 62, f. 37ra-b; pour Alphonse voir, à côté des textes cités à la note précédente, aussi q. 6 , col. 216-241).

${ }_{13}$ Ms. Troyes, BM 62, ff. 37vb-38rb. Dans cette section du texte se trouvent deux des trois citations explicites que Pierre fait de John Rodington : la première concerne la distinction entre l'aspect surnaturel de la béatitude, lié à la grâce indispensable à l'homme pour y parvenir, et l'aspect naturel, lié à la capacité que l'homme a de la recevoir. La deuxième, tout de suite après, concerne le thème de la liberté de la volonté dans l'amour de Dieu, thème sur lequel Pierre ne se laisse aller ni en faveur ni contre l'opinion du maître anglais (ff. 38ra-b). Rodington est cité une troisième fois, mais très rapidement, dans le premier doute après la discussion sur les trois conclusions, doute qui concerne les différents degrés possible de béatitude, où nous trouvons aussi un autre auteur anglais que Pierre nomme dans cette question, à savoir Robert Holkot (f. 38vb). 
surtout, probablement, partagée par les maîtres parisiens de son époque (à l'exception peut-être de Jean de Mirecourt), Pierre semble considérer cet aspect du problème très sereinement.

En ce qui concerne la troisième conclusion de sa question, selon laquelle la fruition béatifique, à proprement parler, revêt de nombreuses facettes et ne peut se réduire à la seule fruition de $\operatorname{Dieu}^{14}$, il faut d'abord rappeler le fait que cela est cohérent avec l'attitude face au bonheur, rappelée ci-dessus ${ }^{15}$; en second lieu, Pierre n'exclut pas la vision intellectuelle de l'ensemble des éléments constituant la fruition béatifique, sans toutefois lui attribuer beaucoup d'importance. II l'évoque très brièvement, sans un grand intérêt manifeste, tout simplement pour rappeler que, dans le cours normal des choses, la béatitude suit la $v^{v i s i o n}{ }^{16}$. En effet Pierre, sur ce point, semble vouloir surtout défendre une position empruntée au troisième livre de la Consolation de Boèce (dont déjà Grégoire de Rimini s'était souvenu en passant ${ }^{17}$ ) et en général aux antiqui, selon laquelle la béatitude serait un status omnium bonorum aggregatione perfectus ${ }^{18}$. Ainsi, il répète plusieurs fois au cours de sa question que la béatitude ne consiste pas seulement dans la fruition et dans le plaisir qui est lié, tout comme elle ne consiste pas dans la vision de Dieu. Elle comprend, en revanche, un certain nombre d'autres éléments nécessaires à la perfection qui la caractérise, au point que l'on pourrait affirmer que la signification du terme beatitudo n'est pas un concept simple, mais qu'il équivaut au contenu d'un long discours résumé par ce seul terme ${ }^{19}$. Parmi ces différents éléments, une importance toute particulière semble toutefois attribuée aux jeux de Pierre, à la sureté subjective de l'immutabilité de la béatitude même, c'est-à-dire à la sureté de la possession d'un état qui, après avoir été acquis, ne peut plus être perdu ou modifié ${ }^{20}$. Mais à propos des éléments constitutifs de la béatitude, en vérité, bien qu'il soit bien clair et déterminé dans son exposée ${ }^{21}$, Pierre semble beaucoup moins attentif et

\footnotetext{
${ }^{14}$ Ms. Troyes, BM 62, f. 37ra-b.

${ }^{15}$ Cfr. note 9.

${ }^{16}$ Ms. Troyes, BM 62, f. 38rb-va.

17 GREgORII ARIMINENSIS OESA Lectura super primum et secundum Sententiarum, ed. D. Trapp / V. Marcolino, t. I, p. 255, II. 18-19 qui se réfère à Boece, De consolatione philosophiae III, 2.
}

${ }^{18}$ Ms. Troyes, BM 62, f. 37rb. n

${ }^{19}$ Ms. Troyes, BM 62, f. 37rb et 38va; voir aussi f. 41vb.

${ }^{20}$ Par exemple voir, dans le ms. Troyes, BM 62, f. 38va.

${ }^{21}$ Il va s'en dire que c'est dans cette section de son exposé que nous trouvons trois des quatre citations explicites que Pierre tire de Robert Holkot : en effet, la première se trouve au même endroit que la dernière de Jean de Rodington dans le premier doute (cf. note 13), tandis que les trois citations suivantes se situent très près les unes des autres dans la discussion sur la troisième conclusion et concernent la signification du verbe desinit, le rôle de la sécurité de la possession immuable de la béatitude dans la définition de la béatitude même et la possibilité que Dieu trompe les bienheureux sur ce point (f. 38va). Donc, à propos des auteurs anglais rappelés explicitement par Pierre dans cette question (cf. aussi note 10), nous pouvons remarquer qu'ils sont mis à cause chacun presque exclusivement à cause 
précis que certains de ses collègues théologiens parisiens de l'époque. En effet, que ce soit dans l'exposé de Grégoire ou dans celui de Alphonse Vargas, qui consacre à ce problème toute sa question 2 et beaucoup $d$ 'autres passages, nous trouvons en effet de longues discussions sur les rapports entre fruitio, dilectio et delectatio dans la vision béatifique ${ }^{22}$; mais Pierre ne semble pas trop vouloir, sur ce point, problématiser davantage, se souvenant alors peut être aussi de son confrère Jean de Mirecourt.

Quelques éléments intéressants pouvant compléter aussi d'une certaine manière le tableau relatif à l'attitude de Pierre sur le problème analysé ici, résident également dans les douze dubia qui suivent l'exposé des trois conclusions : on peut évoquer en particulier le onzième doute, où Pierre se demande si tous les hommes désirent la béatitude ${ }^{23}$. II s'agit d'un cas où, contrairement au précédent, nous pouvons percevoir la distance qui sépare parfois Pierre de Jean de Mirecourt, en comparant ce doute de Pierre à l'ample déroulement de la question 16 du commentaire de Jean, lequel se demande si la fruition de Dieu peut être considérée comme un but de la nature humaine en tant que telle ${ }^{24}$. Jean établit d'abord, à travers I'une de ses discussions dialectiques typiques, que Dieu ne peut pas être ce vers quoi tend le désir naturel de l'homme, parce que l'homme, par nature, désire avant tout la conservation de soi-même ${ }^{25}$. Mais, si l'on déplace le discours du désir exclusivement naturel vers celui qui est libre et rationnel, le but de l'homme peut être alors aussi autre que soi-même, à savoir qu'il peut aussi désirer contempler la vérité26. Jean distingue donc un but ultime de l'homme lié à sa nature d'un but ultime de l'homme relatif à sa raison et à sa liberté, mais semble aussi exclure l'idée que, sans précisions ni exceptions, la fruition de Dieu pourrait être considérée, à n'importe quel titre, un but naturel de l'homme.

Or, par rapport à toute la longue discussion de Jean, dialectiquement très serrée, l'exposé de Pierre est assurément différent. En effet, Pierre explique ${ }^{27}$ que tous désirent naturellement ce qui est bien, et donc la béatitude qui représente pour la créature rationnelle la plus haute forme de bien. Cette conclusion rapide et non problématique s'appuie encore une fois sur l'autorité des antiqui, parmi lesquels Boèce a, comme

d'un thème spécifique, comme si Pierre avait choisi d'utiliser leurs propos seulement sur les points qu'il considérait les plus intéressants et significatifs de leurs exposés.

${ }^{22}$ GregoRII ARIMINENSIS OESA Lectura super primum et secundum Sententiarum, ed. D. Trapp / V. Marcolino, t. I, pp. 217-239; ALPHONSI VARGAS TOLETANI OESA In primum Sententiarum, ed. Venetiis 1490, q. 2, col. 155-161.

${ }^{23}$ Petrus de Ceffona, In IV libros Sententiarum, ms. Troyes, BM 62, f. 42ra-b.

${ }^{24}$ IOANNES DE MIRECURIA, In IV libros Sententiarum in PARODI 1978, pp. 76-90.

${ }^{25}$ IOANneS de MireCURIA, In IV libros Sententiarum in PARodi 1978, pp. 79-82.

${ }^{26}$ IOANNES DE MIRECURIA, In IV libros Sententiarum in PAROdI 1978, pp. 86-88.

27 Petrus de Ceffona, In IV libros Sententiarum, ms. Troyes, BM 62, f. 42ra-b. 
toujours, une place prépondérante, car il nous explique que les hommes se trompent moins sur la nature du but naturel de la béatitude même que sur les voies à suivre pour atteindre la béatitude ${ }^{28}$. Ensuite viennent Augustin et Anselme mais aussi Thomas d'Aquin ${ }^{29}$ qui affirme que toutes les créatures conservent une empreinte de la bonté de Dieu les inclinant à revenir vers cette même bonté, chose que peuvent faire seulement les créatures rationnelles et celles qui possèdent cette béatitude ${ }^{30}$. Donc, s'il est vrai que Pierre s'esquive très vite et sans trop de questions de la discussion autour de ce problème, on peut remarquer qu'il choisit alors des repères très différents par rapport à ce qu'il fait à d'autres endroits ${ }^{31}$, des repères empruntés cette fois à la période des antiqui.

II reste pourtant encore un dernier aspect sur lequel les dubia nous permettent de réfléchir : nous savons que Pierre manifeste un grand intérêt pour les recherches et les thèmes des calculatores d'Oxford et, plus généralement, pour les problèmes typiques de la pensée anglaise des années 1320-3032, au point que, selon des études plus récentes de Kevin Dermott Smith, Ceffons a été probablement le premier à introduire à Paris les écrits de Heytesbury et de Swineshead ${ }^{33}$. Aussi la question qui nous intéresse contient-t-elle en effet des traces évidentes de cet intérêt, même si elle ne nomme pas explicitement les auteurs de ce courant anglais (tandis que, dans les questions précédentes relatives à la première distinction des Sentences, Pierre avait cité à plusieurs reprises le nom de Kilvington ${ }^{34}$ ). Ainsi le dernier argument dans la discussion dialectique de la troisième conclusion présente, par exemple, une analyse de la signification du verbe desinit exprimant le doute que les bienheureux puissent eux aussi cesser de rester dans l'état de la béatitude ${ }^{35}$. Mais ce n'est pas tout: l'auteur, dans le quatrième doute, s'interroge sur la possibilité d'augmenter la béatitude des saints et finit par relier le thème de l'augmentation, autre lieu commun des recherches des calculatores, à la possibilité de penser la fruitio beatifica de plusieurs façons différentes,

\footnotetext{
${ }^{28}$ Ce passage de Boèce suit immédiatement celui qui a été rappelé à la note 17.

${ }^{29}$ En effet, dans cette question, Thomas et Jean Duns Scot sont assez souvent cités en tant que réprésentants de l'état précédent de la discussion: voir par exemple Petrus de CeffonA, In IV libros Sententiarum, ms. Troyes, BM 62, ff. 37ra, $37 v a, 38 r a, 40 r a, 40 v a-b, 42 r a-b, 42 v a$.

${ }^{30}$ Le renvoi est au quatrième livre du commentaire aux Sentences de Thomas, d. 49, q. 1, a. 3.

${ }^{31}$ Voir notes 4, 10, 11, 13, 21.

${ }^{32}$ MURDOCH 1978.

${ }^{33}$ SMITH 1990, p. 349 note 2.

${ }^{34}$ Voir par exemple Petrus de CeffonA, In IV libros Sententiarum, ms. Troyes, BM 62, ff. 35vb et 36ra-b.

${ }^{35}$ Ms. Troyes, BM 62, f. 38rb-va.
} 
rationnellement comparables, comme quelque chose $d^{\prime}$ infini ${ }^{36}$. Et il poursuit dans le doute suivant ce genre de recherches logiques, puisqu'il pose le problème de savoir si, vu le degré de gloire qui comble une âme, il était possible d'atteindre un degré majeur, en faisant appel, encore une fois et assez longuement, aux discussions sur la comparabilité entre les infinis, concluant qu'on peut résoudre la question uniquement $d^{\prime}$ une façon probable, jamais définitive ${ }^{37}$. Ce même ordre de problèmes revient encore dans le sixième doute où il s'interroge sur la possibilité que l'âme puisse recevoir une joie infinie ${ }^{38}$.

L'ampleur des lectures, l'éclectisme et le soin que manifeste leur utilisation, mais encore une certaine prudence doctrinale, me semblent donc les aspects les plus remarquables de cette question. Ainsi, même s'il ne semble pas être parvenu à changer le cours de la pensée de son époque, tout du moins pour ce qui est des textes connus jusqu'à présent, Pierre de Ceffons demeure un témoin significatif de la vie intellectuelle à Paris vers la moitié du siècle, des croisements de doctrines et de personnes. Parce que son esprit attentif, curieux et sensible aux problèmes spéculatifs de l'époque y a participé, il mérite assurément, encore aujourd'hui, notre attention.

\section{Bibliographie finale}

Manuscrits:

Petrus de Ceffona, In IV libros Sententiarum, ms. Troyes, Bibliothèque Municipale 62.

IOANNES DE MIRECURIA, In I librum Sententiarum, ms. Torino, Biblioteca Nazionale Universitaria D. IV. 28.

Autres sources fondamentales:

ALPHONSI VARGAS TOLETANI OESA In primum Sententiarum, edidit T. de Spilimbergo OESA, Paganinus de Paganiniis, Venetiis 1490.

GREGORII ARIMINENSIS OESA Lectura super primum et secundum Sententiarum, ed. D. Trapp / V. Marcolino, t. I, Walter de Gruyter, Berlin-New York 1981.

\footnotetext{
${ }^{36}$ Ms. Troyes, BM 62, f. 39rb.

${ }^{37}$ Ms. Troyes, BM 62, f. 39va.

${ }^{38}$ Ms. Troyes, BM 62, f. 39va-b.
} 
HUgolinI DE URBe VeterI OESA Commentarius in quattuor libros Sententiarum, ed. W. Eckermann, t. I, Augustinus-Verlag, Würzburg 1980.

IOANNES de MiReCURIA, In IV libros Sententiarum, I, 13-16 in M. PAROdI, Questioni inedite tratte dal I libro del commento alle Sentenze di Giovanni di Mirecourt (qq. 13-16), in Medioevo, 3 (1977), pp. 237-284; 4 (1978), pp. 59-92.

Littérature secondaire:

F. Bottin, La scienza degli occamisti. La scienza tardo-medievale dalle origini del paradigma nominalista alla rivoluzione scientifica, Maggioli, Rimini 1982.

A. CORBINI, Una Quaestio de docta ignorantia di Pietro di Ceffons ?, in A. DALL'IGNA / D. RobeRI (éds), Cusano e Lebniz. Prospettive filosofiche, Mimesis, Milano-Udine 2013, pp. 183-202.

J.-F. Genest, Pierre de Ceffons et l'hypothèse du Dieu trompeur, in Z. KALUZA / P. Vignaux (éds), Preuve et raisons à I'Université de Paris: logique, ontologie et théologie au XIVe siècle, Vrin, Paris 1984, pp. 197-214.

E. Mazet, Pierre Ceffons et Oresme - Leur relation revisitée, in S. CAROtI / J. CeleYRetTe (éds), Quia inter doctores est magna dissensio. Les débats de philosophie naturelle à Paris au XIVe siècle, Olschki, Firenze 2004, pp. 175-194.

J.-E. MURDOCH, Subitilitates Anglicanae in Fourteenth-Century Paris: John of Mirecourt and Peter Ceffons, in M. Pelner Cosman / B. Chandler (eds), Machaut's World: Science and Art in the Fourteenth Century, New York Academy of Sciences, New York 1978 (Annals of the New York Academy of Sciences, 314), pp. 51-86.

M. E. REINA, Cognizione intuitiva ed esperienza interiore in Adamo Wodeham, in Rivista di storia della filosofia, 41 (1986), pp. 19-49 et 211-244.

K.-D. SMITH, Theories of Motion, Time and Place in Mid-Fourteenth Century France: Gregory of Rimini, Hugolinus of Orvieto, and Peter Ceffons of Clairvaux, A thesis submitted in partial fulfillment of the requirements for the degree of Doctor of Philosophy (History), University of Wisconsin-Madison 1990.

K. TACHAU, Vision and Certitude in the Age of Ockham. Optics, Epistemology and the Foundations of Semantics 1250-1345, E. J. Brill, Leiden-New York-København-Köln 1988.

D. TRAPP, Peter Ceffons of Clairvaux, in Recherches de Théologie ancienne et médiévale, 24 (1957), pp. 101154. 
ID., A Round-Table Discussion Between a Parisian OCist-Team and OESA-Team about AD 1350, in Recherches de théologie ancienne et médiévale, 51 (1984), pp. 206-222. 